\title{
Functional Larynx Preservation in Patients With Locally Advanced Squamous Cell Carcinoma of the Larynx and Hypopharynx Treated With Induction Chemotherapy vs. Concurrent Chemoradiation Alone
}

\author{
Anthony D. Nehlsen ${ }^{1}$, Eric J. Lehrer ${ }^{2}$, Daniel R. Dickstein ${ }^{3}$, Marshall R. Posner ${ }^{4}$, Krzysztof Misiukiewicz ${ }^{4}$ \\ , Jerry Liu ${ }^{3}$, Vishal Gupta ${ }^{1}$, Richard L. Bakst ${ }^{1}$, Sonam Sharma ${ }^{2}$ \\ 1. Radiation Oncology, Mount Sinai, New York, USA 2. Radiation Oncology, Icahn School of Medicine at Mount Sinai, \\ New York City, USA 3. Radiation Oncology, Icahn School of Medicine at Mount Sinai, New York, USA 4. Medical \\ Oncology, Icahn School of Medicine at Mount Sinai, New York, USA
}

Corresponding author: Anthony D. Nehlsen, anthonynehlsen7@gmail.com

\section{Abstract \\ Objectives}

Chemoradiation therapy (CRT) has been established as a standard treatment for locally advanced hypopharynx/larynx squamous cell carcinoma (SCC) but the role of induction chemotherapy (IC) remains unclear. The primary outcome of this study is to determine whether functional larynx-preservation survival (FLPS) is improved with the addition of IC in these patients. Secondary outcomes were overall survival (OS), progression-free survival (PFS), distant metastasis-free survival (DMFS), and laryngectomy rates.

\section{Methods}

Records for patients with AJCC 8th edition clinical stage III-IVB laryngeal and hypopharyngeal SCC treated with CRT +/- IC from 2005-2019 were reviewed. FLPS was defined as time until death, progression, laryngectomy, or non-functional larynx. Kaplan-Meier curves were generated for FLPS, OS, PFS, and DMFS. Outcomes were compared using the stratified log-rank test. Laryngectomy rates were compared using Fisher's exact test.

\section{Results}

We included 52 patients with laryngeal and 38 with hypopharyngeal SCC $(n=90) ; 19$ patients with laryngeal SCC and 19 with hypopharyngeal SCC received IC (median three cycles). There were no differences in the three-year FLPS ( $61 \%$ vs $67.8 \%$; $p=0.88$ ), OS ( $73.9 \%$ vs $86.2 \%$; $p=0.42$ ), PFS ( $53.6 \%$ vs $62.6 \%$; $p=0.44$ ), or DMFS $(65.2 \%$ vs $71.5 \%, p=0.85)$ between patients who did and did not receive IC all patients. Laryngectomy rates did not differ with and without IC (18.4\% vs $7.7 \%$; $\mathrm{p}=0.19)$.

Review began 06/14/2021 Review ended 06/29/2021 Published 07/11/2021

๑) Copyright 2021 Nehlsen et al. This is an open access article distributed under the terms of the Creative Commons Attribution License CC-BY 4.0., which permits unrestricted use, distribution, and reproduction in any medium, provided the original author and source are credited.

\section{Conclusion}

In this study of advanced laryngeal and hypopharyngeal SCC, IC did not improve three-year FLPS, OS, PFS, or laryngectomy rates compared to CRT alone. A large prospective series would provide a more robust understanding of the role of IC in this group of patients.

Categories: Otolaryngology, Radiation Oncology, Oncology

Keywords: larynx preservation, hypopharynx, radiation, squamous cell carcinoma, induction chemotherapy

\section{Introduction}

In addition to local control and survival outcomes, optimizing functional organ preservation in patients with locally advanced laryngeal and hypopharyngeal squamous cell carcinomas (SCC) continues to be an important outcome measure due to the improved quality of life measures associated with larynx preservation [1]. Laryngectomy is associated with significant morbidity, including the development of difficulties with speech and swallowing, and offers no benefit in terms of overall survival (OS) compared to concurrent chemoradiation therapy (CRT) for many patients [2-4]. It is important to note, however, that laryngectomy (+/- adjuvant therapy) can improve local control and possibly OS in patients with T4a primary lesions $[5,6]$. In patients without T4a disease, high rates of local control and larynx preservation are possible with the use of CRT, and those who do experience local failures can often be effectively salvaged with surgery [7]. Numerous clinical trials have analyzed the role of CRT to improve larynx preservation rates, with both induction chemotherapy (IC) followed by radiation and CRT being used. 
The Veteran Affairs Larynx Trial established the role of combined modality therapy in the treatment of locally advanced laryngeal cancer, showing equivalent OS and a 64\% laryngeal preservation rate at two years in patients receiving IC followed by radiation therapy (RT) compared to those receiving laryngectomy with postoperative RT [2]. This was further examined in hypopharyngeal SCC by EORTC trial 24891, which also demonstrated a $22 \%$ rate of survival with an intact larynx at five years and similar rates of local failure, OS, and progression-free survival (PFS) in the IC plus RT arm compared to the group receiving up-front surgery [8]. Intensified IC in SCC of the larynx and hypopharynx using docetaxel, cisplatin, and 5-FU (TPF) compared to cisplatin and 5-FU (PF) has also been studied, with randomized data demonstrating improvements in larynx preservation rates and OS $[9,10]$.

Definitive CRT has also been studied in the randomized setting, with RTOG 91-11 showing improved local control and larynx preservation at 10 years compared to sequential chemotherapy and RT or RT alone, with similar rates of OS [3]. Additionally, combining CRT and induction therapy has emerged as a treatment option. The TREMPLIN trial, which randomized patients with stage III-IV laryngeal or hypopharyngeal SCC to CRT with cisplatin or cetuximab after induction with three cycles of TPF resulted in excellent rates of larynx preservation (87\% vs $82 \%$, respectively) [11].

Despite the improvement in outcomes seen in the trials above, conflicting studies have not shown a significant benefit to induction chemotherapy when compared to CRT alone. For example, the PARADIGM and DeCIDE trials, each of which included a significant number of patients with SCC of the larynx and hypopharynx, showed no benefit to the use of IC when compared to CRT alone [12, 13]. Similarly, the MACHNC meta-analysis found improved outcomes with CRT when compared to IC followed by RT [14].

This data, in combination with the significantly higher rates of treatment-related toxicity and complexity seen in patients undergoing IC unless treated in experienced multi-disciplinary centers, makes the routine use of IC in the treatment of SCC of the larynx and hypopharynx a topic of ongoing discovery [9-13]. In this study, we aim to compare the rates of functional-larynx preservation survival in patients with stage III-IV SCC of the larynx and hypopharynx treated with IC followed by CRT to those patients receiving CRT alone using a retrospective cohort of patients treated at a large academic medical center.

\section{Materials And Methods}

This study was approved by the Mount Sinai Institutional Review Board. We identified the records of patients with locally advanced clinically staged III-IV (AJCC 8th edition) SCC of the larynx and hypopharynx treated at our institution from 2005 through 2018. Eligible patients were at least 18 years of age, had pathologic confirmation of SCC of the larynx or hypopharynx, with localized disease at presentation, and were treated with multi-agent IC (TPF or PF) followed by definitive CRT or with definitive CRT alone. Charts were reviewed for radiation, chemotherapy, staging, pathologic, demographic, and outcomes data.

The aim of this study was to compare treatment outcomes in patients treated with IC followed by definitive CRT to patients treated with CRT alone when using laryngeal preservation for locally advanced larynx and hypopharynx SCC. The primary endpoint was functional larynx-preservation survival (FLPS), which was defined as the time until death, local progression, laryngectomy, or non-functional larynx. Secondary endpoints were OS, PFS, and rates of laryngectomy at the last follow-up. Response to IC was measured using computed tomography or positron emission tomography (PET) imaging based on the studies available.

Statistical analyses were conducted using R Studio ver 1.1.419 (R Studio, Boston, MA). The 'ggplot2' package ver 3.2.1 was used to generate Kaplan-Meier curves and log-rank tests. The 'survival' package ver 3.1-8 was used to conduction the cox proportional hazards models. Each time-to-event outcome (OS, PFS, and FLPS) was stratified by receipt of induction chemotherapy and then by disease subsite (larynx and hypopharynx). The stratified log-rank test was used to compare these outcomes by the aforementioned groupings, where the null hypothesis was rejected for $\mathrm{p}<0.05$. Univariate and multivariate cox proportional hazards models were used to assess the impact of covariates on OS, PFS, and FLPS. We included T/N stage, smoking status, and receipt of induction chemotherapy in the multivariate analysis due to the clinical behavior of SCC of the larynx and hypopharynx. We included any covariates with a p-value of 0.3 on the univariate analysis in the multivariate analysis. Goodness of fit of the multivariate model was assessed using the log-rank test, where a backward selection method was used to determine the optimal multivariate model. Satisfaction of the proportional hazards assumption was assessed using Schoenfeld's Test and visual inspection of Schoenfeld Residuals.

\section{Results}

A total of 90 patients meeting our inclusion criteria were identified: 52 of these patients had SCC of the larynx, of which 19 received IC and 33 were treated with CRT alone. Additionally, 38 patients had SCC of the hypopharynx, of which 19 received IC, and 19 were treated with CRT alone. The median follow-up for the cohort was 24 months (range 2-121). Patient characteristics are further detailed in Table 1. As shown in Table 2, the two cohorts were well matched for T stage, smoking history, primary site of disease, ECOG performance status, and age. However, there were significantly more patients with N2 or N3 disease $(p=0.009)$ and clinical stage IV disease $(p=0.0001)$ in the IC chemotherapy group. 


\section{Cureus}

\begin{tabular}{|c|c|c|}
\hline & Induction $(\mathrm{n}=38)$ & Non-Induction $(n=52)$ \\
\hline Age (median) & 63 & 66 \\
\hline \multicolumn{3}{|l|}{ Gender } \\
\hline Male & 28 (73.7\%) & 45 (86.5\%) \\
\hline Female & 10 (26.3\%) & 7 (13.5\%) \\
\hline \multicolumn{3}{|l|}{ Ethnicity } \\
\hline White & 25 (65.8\%) & $23(44.2 \%)$ \\
\hline African American & $8(21.1 \%)$ & $13(25.0 \%)$ \\
\hline Other & 5 (13.1\%) & $16(30.8 \%)$ \\
\hline \multicolumn{3}{|l|}{ ECOG } \\
\hline 0 & $6(18.2 \%)$ & $13(26.0)$ \\
\hline 1 & 24 (72.7\%) & $26(52.0 \%)$ \\
\hline$\geq 2$ & $3(9.1 \%)$ & $11(22.0 \%)$ \\
\hline \multicolumn{3}{|l|}{ Smoking history } \\
\hline Yes & 31 (81.6\%) & 44 (84.6\%) \\
\hline No & $7(18.4 \%)$ & 8 (15.4\%) \\
\hline
\end{tabular}

\section{TABLE 1: Patient Demographics}

\begin{tabular}{|c|c|c|c|}
\hline & Induction $(n=38)$ & Non-Induction $(n=52)$ & p-Value \\
\hline \multicolumn{4}{|l|}{ Primary site } \\
\hline Larynx & 19 (50.0\%) & $33(63.5 \%)$ & \multirow{2}{*}{0.28} \\
\hline Hypopharynx & 19 (50.0\%) & 19 (36.5\%) & \\
\hline \multicolumn{4}{|l|}{ T Stage } \\
\hline T1-3 & $25(69.4 \%)$ & $43(82.7 \%)$ & \multirow{2}{*}{0.20} \\
\hline T4 & $11(30.6 \%)$ & $9(17.3 \%)$ & \\
\hline \multicolumn{4}{|l|}{ N Stage } \\
\hline N0-1 & $12(33.3 \%)$ & $33(63.5 \%)$ & \multirow{2}{*}{0.009} \\
\hline N2-3 & $24(66.7 \%)$ & $19(36.5 \%)$ & \\
\hline \multicolumn{4}{|c|}{ AJCC Clinical Stage } \\
\hline III & $5(13.2 \%)$ & 27 (51.9\%) & \multirow{2}{*}{0.0001} \\
\hline IV & $33(86.8 \%)$ & $25(48.1 \%)$ & \\
\hline
\end{tabular}

\section{TABLE 2: Tumor Characteristics}

The median radiation dose received was $70 \mathrm{~Gy}$ (range 60-75) in 35 fractions (range 30-50). A median of three cycles (range 1-4) of chemotherapy were given in those receiving IC. Twenty-seven patients were treated with TPF and 11 patients were treated with PF.

FLPS rates at three years did not differ significantly between the patients receiving IC and those receiving CRT alone for the entire cohort (Figure $1 \mathrm{~A}$ ), with rates of $61 \%$ and $67.8 \%$ respectively $(\mathrm{p}=0.48$ ). Similar 


\section{Cureus}

findings were seen on subset analysis by primary tumor site for those with SCC of the larynx (52.6\% vs $68.8 \% ; \mathrm{p}=0.22$ ) and hypopharynx (66.9\% vs $65.3 \%$; $\mathrm{p}=0.58)$. These findings are shown in Figure $1 B$ and Figure $1 C$, respectively.

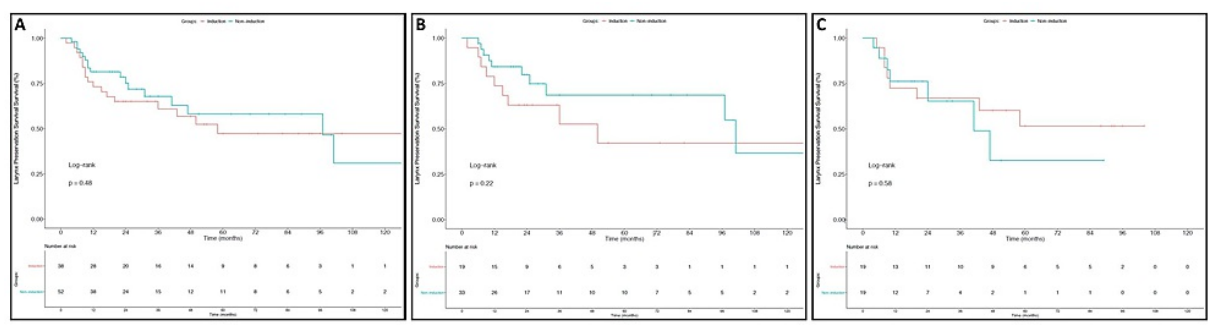

\section{FIGURE 1: Functional Larynx Preservation Survival}

This figure depicts functional larynx preservation survival (FLPS) outcomes for all patients (A), larynx SCC patients (B), and hypopharynx SCC patients (C). Outcomes for patients who did not receive induction chemotherapy are in blue and those who did receive induction chemotherapy are in red.

Rates of three-year OS were also similar in the entire cohort for patients treated with IC and those treated with CRT alone (Figure $2 A$ ) $(73.9 \%$ vs $86.2 \%$; $\mathrm{p}=0.42)$. As shown in Figures $2 B-2 C$ respectively, there were also no significant differences observed in three-year OS for larynx ( $76 \%$ vs $86.3 \%$; $\mathrm{p}=0.36$ ) or hypopharynx patients $(70.8 \%$ vs $86.7 \%$; $\mathrm{p}=0.96)$. Additionally, PFS at three years did not differ between patients who did and did not receive IC for the entire cohort (Figure $3 A$ ) $(53.6 \%$ vs $62.6 \%$; $\mathrm{p}=0.44)$ or on subset analysis for patients with SCC of the larynx (Figure 3B) (57.9\% vs 61.8\%; $\mathrm{p}=0.29$ ) or hypopharynx (Figure 3C) (50\% vs $63.6 \%$; $=0.99)$. Finally, as shown in Figure $4 A$, three-year DMFS rates for all patients did not differ between those who did and did not receive IC ( $65.2 \%$ vs $71.5 \%, p=0.85)$. As depicted in Figures $4 B-4 C$, this was also true for patients with laryngeal SCC ( $75.2 \%$ vs $66.3 \%$, $\mathrm{p}=0.93)$ and hypopharyngeal SCC $(55.2 \%$ vs $82.9 \%$, $\mathrm{p}=0.76)$.

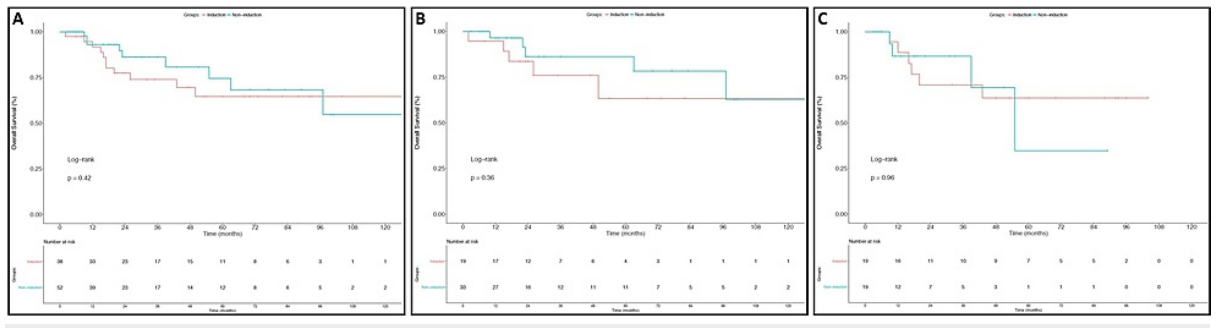

FIGURE 2: Overall Survival

This figure depicts overall survival (OS) outcomes for all patients (A), larynx SCC patients (B), and hypopharynx SCC patients (C). Outcomes for patients who did not receive induction chemotherapy are in blue and those who did receive induction chemotherapy are in red.

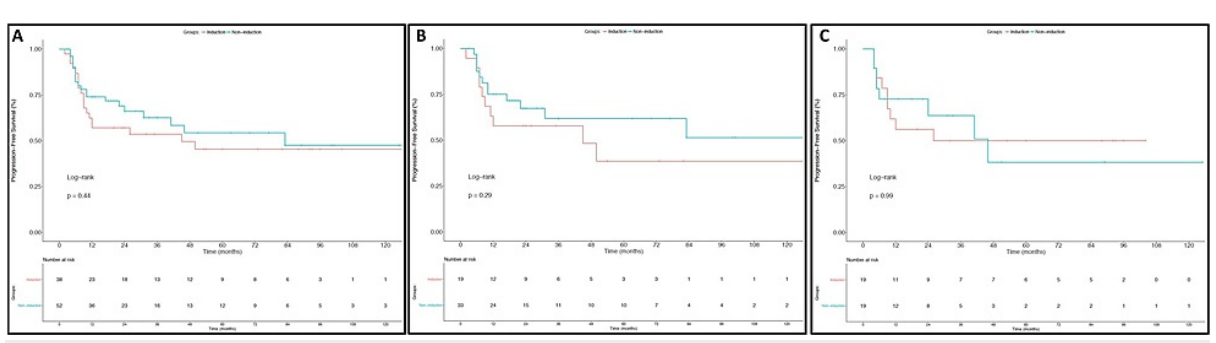

\section{FIGURE 3: Progression-Free Survival}

This figure depicts progression-free survival (PFS) outcomes for all patients (A), larynx SCC patients (B), and hypopharynx SCC patients (C). Outcomes for patients who did not receive induction chemotherapy are in blue and those who did receive induction chemotherapy are in red. 


\section{Cureus}

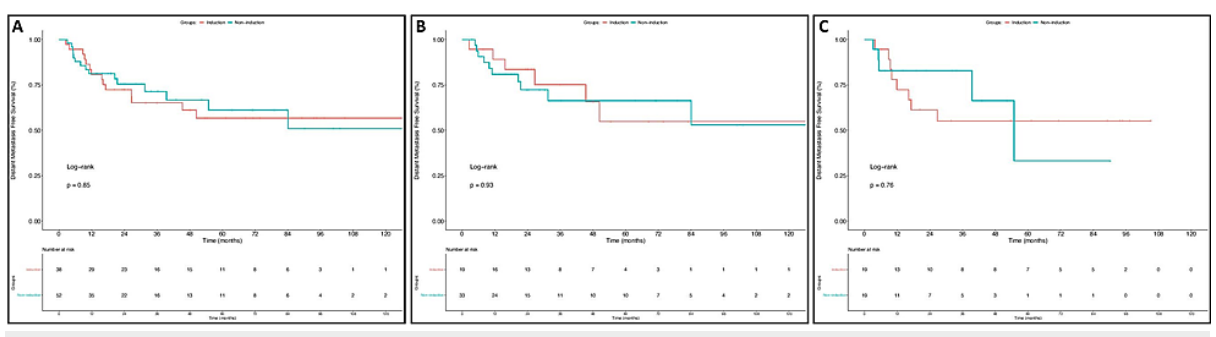

\section{FIGURE 4: Distant Metastasis-Free Survival}

This figure depicts distant metastasis-free survival (DMFS) outcomes for all patients (A), larynx SCC patients (B), and hypopharynx SCC patients (C). Outcomes for patients who did not receive induction chemotherapy are in blue and those who did receive induction chemotherapy are in red.

Laryngectomy rates are shown in Table 3 . In the entire cohort, 11 patients (12.2\%) required laryngectomy, with seven (18.4\%) occurring in the IC group compared to $3(7.7 \%)$ in the patients treated with CRT alone $(\mathrm{p}=0.19)$. In the larynx and hypopharynx subgroups, these rates were $21.1 \%$ vs $6.1 \%(\mathrm{p}=0.18)$ and $15.8 \%$ vs $10.5 \%$ ( $>0.99$ ), respectively. Laryngectomy was performed for recurrence in $5 / 7$ (71\%) patients in the IC group (with the other two being for a non-functional larynx) and in all four patients in the CRT alone group.

\begin{tabular}{|c|c|c|c|}
\hline & Induction $(n=38)$ & Non-Induction $(n=52)$ & p-Value \\
\hline Entire cohort & $7(18.4 \%)$ & $4(7.7 \%)$ & 0.19 \\
\hline Larynx & $4(21.1 \%)$ & $1(6.1 \%)$ & 0.18 \\
\hline Hypopharynx & $3(15.8 \%)$ & $3(10.5 \%)$ & $>0.99$ \\
\hline
\end{tabular}

TABLE 3: Salvage Laryngectomy Rates

On multivariate analysis (MVA) (Table 4 for FLPS), induction chemotherapy was not associated with improved FLPS, OS, or PFS ( $\mathrm{p}>0.05$ ). N2 disease was associated with worse outcomes for FLPS (HR 8.56, 95\% CI 1.63-45.01, p=0.01), OS (HR: 3.57, 95\% CI: 1.22-10.45, p=0.02), PFS (HR 3.76, 95\% CI 1.44-9.77, $\mathrm{p}=0.007$ ), and DMFS (HR: 16.32, 95\% CI: 1.20-222.26, p=0.04). N3 disease was also associated with worse outcomes for FLPS (HR 41.4, 95\% CI 5.19-330.34, p <0.001), OS (HR 18.35, 95\% CI 2.13-157.73, $\mathrm{p}=0.008$ ), PFS (HR 24.8, 95\% CI 4.56-135.03, p<0.001), and DMFS (HR: 269.58, 95\% CI: 11.46-6,341.03, p<0.001). There was no difference in three-year FLPS ( $63.6 \%$ vs $47.2 \%$, $\mathrm{p}=0.93)$, OS ( $61 \%$ vs $81.6 \%$, $\mathrm{p}=0.85$ ), or PFS ( 33.5 vs $37.9 \%, \mathrm{p}=0.48$ ) in patients with N2-3 disease receiving IC compared to those who did not, respectively. 


\section{Cureus}

\begin{tabular}{|c|c|c|c|}
\hline Variable & Hazard Ratio (HR) & $95 \% \mathrm{Cl}$ & p-Value \\
\hline Age & 0.98 & 0.94-1.02 & 0.33 \\
\hline \multicolumn{4}{|l|}{ Induction } \\
\hline Yes & Ref & Ref & Ref \\
\hline No & 0.88 & $0.32-2.46$ & 0.81 \\
\hline \multicolumn{4}{|l|}{ Smoking } \\
\hline No & Ref & Ref & Ref \\
\hline Yes & 3.3 & $0.31-34.70$ & 0.32 \\
\hline \multicolumn{4}{|l|}{ T Stage } \\
\hline 1 & Ref & Ref & Ref \\
\hline 2 & 0.34 & $0.03-4.50$ & 0.41 \\
\hline 3 & 0.99 & $0.09-11.37$ & 0.99 \\
\hline 4 & 1.11 & $0.10-12.81$ & 0.94 \\
\hline \multicolumn{4}{|l|}{ N Stage } \\
\hline 0 & Ref & Ref & Ref \\
\hline 1 & 2.66 & $0.40-17.75$ & 0.31 \\
\hline 2 & 8.56 & $1.63-45.01$ & 0.01 \\
\hline 3 & 41.4 & $5.19-330.34$ & $<0.001$ \\
\hline
\end{tabular}

\section{TABLE 4: Multivariate Analysis for FLPS}

FLPS: functional larynx-preservation survival

Response to IC was available in 25 patients, with one (4\%) patient demonstrating progression of disease, 20 (80\%) showing a partial response (PR), and four (16\%) exhibiting a complete response (CR). For the larynx SCC subset, 11 patients demonstrated a PR and two exhibited a CR. One patient in the hypopharynx subset suffered from POD while nine experienced a PR and two experienced a CR.

\section{Discussion}

In our study population, the use of IC did not improve FLPS, OS, PFS, or DMFS in patients treated with definitive CRT for locally advanced SCC of the larynx or hypopharynx. While patients in the IC group did have a higher proportion of patients with N2/N3 disease, which was associated with higher rates of failure, multivariate analysis did not demonstrate any benefit to IC in our cohort of patients. However, the imbalance of nodal disease burden between the two groups remains a confounding variable that could have influenced the results. Additionally, clinical trials, such as TREMPLIN and GORTEC 2000-01, demonstrated high rates of functional larynx preservation in patients with stage III/IV laryngeal or hypopharyngeal SCC receiving induction chemotherapy, suggesting that there may be a subset of patients who would significantly benefit from the use of IC prior to definitive CRT $[10,11]$.

Therefore, the implementation of strategies aimed at identifying those patients at the highest risk of local failure may help guide the use of IC. Due to the high propensity for local failure requiring laryngectomy seen in patients with T4 tumors in the VA Larynx Trial, $\mathrm{T}$ stage has since been viewed as a strong predictor for local failure after CRT [2]. However, in our analysis, T stage was not significantly linked to lower rates of FLPS, OS, PFS, or DMFS. Other studies have also found no link between T stage and local recurrence rate, finding instead that primary tumor volume may be a better predictive tool [15-18]. Our study showed a strong correlation between advanced nodal status (N2-3) and outcomes, which has been corroborated by a number of studies finding links between nodal stage and volume with survival and control outcomes $[15,18$, 19]. Finally, there is additional data supporting the use of PET imaging, including metabolic tumor volume and maximum SUV, as a predictor for larynx preservation and OS [20,21]. Although additional studies are needed to validate these findings, risk-stratifying patients based on primary tumor volume, the extent of nodal involvement, and PET findings may provide a better means of identifying those that may benefit from 
Identifying patients who are likely to tolerate and respond to IC is an important avenue of future research. Evidence by Liu et al. suggests that patients with advanced SCC of the hypopharynx who completely respond to induction chemotherapy have excellent rates of larynx preservation and survival. However, those treated with larynx preservation who achieved a partial response or less did markedly worse in terms of both OS and larynx preservation [22]. While our cohort lacked the statistical power for a similar type of analysis, this may represent a method for the identification of patients who may benefit from more aggressive treatment options or surveillance schedules. Other studies of organ preservation protocols using induction chemotherapy in both laryngeal and hypopharyngeal SCC have also found improved survival and organ preservation outcomes in patients with significant responses to IC compared to those with partial response [23, 24]. This suggests that the selection of patients likely to have significant responses to chemotherapy may allow for the identification of patients with locally advanced disease that are most likely to achieve long-term survival with meaningful larynx preservation. One such strategy by Wichmann et al. used PET and endoscopic evaluation after one cycle of multi-agent induction chemotherapy to prospectively create a prognostic scoring system that predicted laryngectomy-free survival and OS in patients undergoing larynx preservation therapy. Their prognostic scoring also aided in the early recognition of non-responders who were more likely to benefit from early salvage laryngectomy [25]. While additional prospective trials are needed to validate these findings as a means of predicting which patients are most likely to respond to IC, the use of prognostic scoring systems provides an exciting area of future study. A large randomized trial (SALTORL), comparing definitive CRT to IC followed by RT in patients locally advanced larynx and hypopharynx SCC, is currently ongoing and will hopefully provide more definitive insight into the role of IC in this setting.

Our study has a number of important limitations. Notably, this was a retrospective analysis, which makes it difficult to account for confounding biases that may have affected our results and limits the ability to determine causation from our findings. Patients in the IC arm had a higher prevalence of stage IV and N2 disease, which could have affected larynx preservation or OS rates, particularly due to the worse prognosis associated with N2 disease. The groups were otherwise well balanced in terms of tumor characteristics. Additionally, the number of cycles and selection of chemotherapeutic agents given to each patient during their induction regimens was not uniform which may have affected disease outcomes.

\section{Conclusions}

In this retrospective analysis of patients with stage III-IV SCC of the larynx and hypopharynx, there was no significant difference between IC followed by CRT compared to CRT alone for our primary outcome of FLPS or in our secondary outcomes of OS, PFS, DMFS and laryngectomy rate. On MVA there was also no improvement in outcomes associated with the use of IC. However, the patients in the IC arm were more likely to have clinical stage IV and N2-3 disease, which could have impacted our results. N2-3 disease was associated with worse FLPS, OS, PFS, and DMFS while T stage was not significantly associated with these outcomes. Additional research aimed at identifying which patients undergoing larynx preservation are at highest risk of local failure and are most likely to benefit from IC is needed to further understand the role of IC in the management of patients with locally advanced cancers of the larynx and hypopharynx.

\section{Additional Information \\ Disclosures}

Human subjects: Consent was obtained or waived by all participants in this study. Mount Sinai IRB issued approval Approval GCO 19-1998; IRB-19-02310. This research was approved by the Mount Sinai IRB with an approval number of IRB-19-02310. Animal subjects: All authors have confirmed that this study did not involve animal subjects or tissue. Conflicts of interest: In compliance with the ICMJE uniform disclosure form, all authors declare the following: Payment/services info: All authors have declared that no financial support was received from any organization for the submitted work. Financial relationships: All authors have declared that they have no financial relationships at present or within the previous three years with any organizations that might have an interest in the submitted work. Other relationships: All authors have declared that there are no other relationships or activities that could appear to have influenced the submitted work.

\section{References}

1. Terrell JE, Fisher SG, Wolf GT: Long-term quality of life after treatment of laryngeal cancer. The Veterans Affairs Laryngeal Cancer Study Group. Arch Otolaryngol Head Neck Surg. 1998, 124:964-71. 10.1001/archotol.124.9.964

2. Wolf GT, Fisher SG, Hong WK, et al.: Induction chemotherapy plus radiation compared with surgery plus radiation in patients with advanced laryngeal cancer. N Engl J Med. 1991, 324:1685-90. 10.1056/NEJM199106133242402

3. Forastiere AA, Zhang Q, Weber RS, et al.: Long-term results of RTOG 91-11: a comparison of three nonsurgical treatment strategies to preserve the larynx in patients with locally advanced larynx cancer. J Clin Oncol. 2013, 31:845-52. 10.1200/JCO.2012.43.6097 
4. Network, N.C.C. Head and neck cancers . (2019). Accessed: July 9, 2021: https://www.nccn.org/professionals/physician_gls/pdf/head-and-neck.pdf.

5. Rosenthal DI, Mohamed AS, Weber RS, et al.: Long-term outcomes after surgical or nonsurgical initial therapy for patients with T4 squamous cell carcinoma of the larynx: A 3-decade survey. Cancer. 2015, 121:1608-19. 10.1002/cncr.29241

6. Grover S, Swisher-McClure S, Mitra N, et al.: Total laryngectomy versus larynx preservation for T4a larynx cancer: patterns of care and survival outcomes. Int J Radiat Oncol Biol Phys. 2015, 92:594-601. 10.1016/j.ijrobp.2015.03.004

7. Rodriguez CP, Adelstein DJ, Rybicki LA, et al.: Clinical predictors of larynx preservation after multiagent concurrent chemoradiotherapy. Head Neck. 2008, 30:1535-42. 10.1002/hed.20902

8. Lefebvre JL, Andry G, Chevalier D, et al.: Laryngeal preservation with induction chemotherapy for hypopharyngeal squamous cell carcinoma: 10-year results of EORTC trial 24891. Ann Oncol. 2012, 23:270814. 10.1093/annonc/mds065

9. Janoray G, Pointreau Y, Garaud P, et al.: Long-term results of GORTEC 2000-01: A multicentric randomized phase III trial of induction chemotherapy with cisplatin plus 5-fluorouracil, with or without docetaxel, for larynx preservation. J Natl Cancer Inst. 2016, 108:djv368. 10.1093/jnci/djv368

10. Lorch JH, Goloubeva O, Haddad RI, et al.: Induction chemotherapy with cisplatin and fluorouracil alone or in combination with docetaxel in locally advanced squamous-cell cancer of the head and neck: long-term results of the TAX 324 randomised phase 3 trial. Lancet Oncol. 2011, 12:153-9. 10.1016/S14702045(10)70279-5

11. Lefebvre JL, Pointreau Y, Rolland F, et al.: Induction chemotherapy followed by either chemoradiotherapy or bioradiotherapy for larynx preservation: the TREMPLIN randomized phase II study. J Clin Oncol. 2013, 31:853-9. 10.1200/JCO.2012.42.3988

12. Haddad R, O'Neill A, Rabinowits G, et al.: Induction chemotherapy followed by concurrent chemoradiotherapy (sequential chemoradiotherapy) versus concurrent chemoradiotherapy alone in locally advanced head and neck cancer (PARADIGM): a randomised phase 3 trial. Lancet Oncology. 2013, 14:257264. 10.1016/S1470-2045(13)70011-1

13. Cohen EE, Karrison TG, Kocherginsky M, et al.: Phase III randomized trial of induction chemotherapy in patients with N2 or N3 locally advanced head and neck cancer. J Clin Oncol. 2014, 32:2735-43. 10.1200/JCO.2013.54.6309

14. Pignon JP, le Maître A, Maillard E, Bourhis J: Meta-analysis of chemotherapy in head and neck cancer (MACH-NC): an update on 93 randomised trials and 17,346 patients. Radiother Oncol. 2009, 92:4-14. 10.1016/j.radonc.2009.04.014

15. Sharrett JM, Ward MC, Murray E, et al.: Tumor volume useful beyond classic criteria in selecting larynx cancers for rreservation therapy. Laryngoscope. 2020, 130:2372-7. 10.1002/lary.28396

16. Romesser PB, Qureshi MM, Subramaniam RM, Sakai O, Jalisi S, Truong MT: A prognostic volumetric threshold of gross tumor volume in head and neck cancer patients treated with radiotherapy. Am J Clin Oncol. 2014, 37:154-61. 10.1097/COC.0b013e31826e04d6

17. Knegjens JL, Hauptmann M, Pameijer FA, et al.: Tumor volume as prognostic factor in chemoradiation for advanced head and neck cancer. Head Neck. 2011, 33:375-82. 10.1002/hed.21459

18. Okazaki E, Kawabe J, Oishi M, et al.: Prognostic significance of pretreatment 18F-fluorodeoxyglucose positron emission tomography evaluation using metabolic tumor volume of the primary tumor and lymph nodes in advanced hypopharyngeal cancer. Head Neck. 2019, 41:739-47. 10.1002/hed.25429

19. Schüttrumpf L, Marschner S, Scheu K, et al.: Definitive chemoradiotherapy in patients with squamous cell cancers of the head and neck - results from an unselected cohort of the clinical cooperation group "Personalized Radiotherapy in Head and Neck Cancer". Radiat Oncol. 2020, 15:7. 10.1186/s13014-019-14524

20. Miyabe J, Hanamoto A, Tatsumi M, et al.: Metabolic tumor volume of primary tumor predicts survival better than T classification in the larynx preservation approach. Cancer Sci. 2017, 108:2030-8. 10.1111/cas.13345

21. Werner J, Hüllner MW, Rupp NJ, Huber AM, Broglie MA, Huber GF, Morand GB: Predictive value of pretherapeutic maximum standardized uptake value (Suvmax) in laryngeal and hypopharyngeal cancer. Sci Rep. 2019, 9:8972. 10.1038/s41598-019-45462-y

22. Liu S, Fang J, Ma H, et al.: Rational choice of induction chemotherapy-based larynx preservation for hypopharyngeal cancer. Acta Otolaryngol. 2018, 138:1146-53. 10.1080/00016489.2018.1506152

23. Bozec A, Benezery K, Ettaiche M, et al.: Induction chemotherapy-based larynx preservation program for locally advanced hypopharyngeal cancer: oncologic and functional outcomes and prognostic factors. Eur Arch Otorhinolaryngol. 2016, 273:3299-306. 10.1007/s00405-016-3919-3

24. León X, Valero C, Rovira C, Rodriguez C, López M, García-Lorenzo J, Quer M: Relationship between response to induction chemotherapy and disease control in patients with advanced laryngeal carcinoma included in an organ preservation protocol. Eur Arch Otorhinolaryngol. 2017, 274:2581-7. 10.1007/s00405017-4548-1

25. Wichmann G, Krüger A, Boehm A, et al.: Induction chemotherapy followed by radiotherapy for larynx preservation in advanced laryngeal and hypopharyngeal cancer: Outcome prediction after one cycle induction chemotherapy by a score based on clinical evaluation, computed tomography-based volumetry and 18F-FDG-PET/CT. Eur J Cancer. 2017, 72:144-55. 10.1016/j.ejca.2016.11.013 